\title{
Anterior ankle arthroscopy, distraction or dorsiflexion?
}

\author{
Peter A. J. de Leeuw · Pau Golanó • \\ Joan A. Clavero $\cdot$ C. Niek van Dijk
}

Received: 12 December 2009/ Accepted: 10 February 2010/Published online: 9 March 2010

(C) The Author(s) 2010. This article is published with open access at Springerlink.com

\begin{abstract}
Anterior ankle arthroscopy can basically be performed by two different methods; the dorsiflexion- or distraction method. The objective of this study was to determine the size of the anterior working area for both the dorsiflexion and distraction method. The anterior working area is anteriorly limited by the overlying anatomy which includes the neurovascular bundle. We hypothesize that in ankle dorsiflexion the anterior neurovascular bundle will move away anteriorly from the ankle joint, whereas in ankle distraction the anterior neurovascular bundle is pulled tight towards the joint, thereby decreasing the safe anterior working area. Six fresh frozen ankle specimens, amputated above the knee, were scanned with computed tomography. Prior to scanning the anterior tibial artery was injected with contrast fluid and subsequently each ankle was scanned both in ankle dorsiflexion and in distraction. A special device was developed to reproducibly obtain ankle
\end{abstract}

P. A. J. de Leeuw $(\bowtie) \cdot$ C. N. van Dijk

Academic Medical Center, Department of Orthopaedic Surgery,

University of Amsterdam, PO Box 22700, 1100 DE Amsterdam,

The Netherlands

e-mail: p.a.deleeuw@amc.uva.nl; p.a.deleeuw@amc.nl

C. N. van Dijk

e-mail: c.n.vandijk@amc.uva.nl

P. Golanó

Laborarory of Arthroscopic and Surgical Anatomy, Department of Pathology and Experimental Therapeutics (Human Anatomy Unit), University of Barcelona, c/Feixa Llarga s/n (Campus Bellvitge),

L'Hospitalet de Llobregat, 08097 Barcelona, Spain

e-mail:pgolano@ub.edu

\section{J. A. Clavero}

Diagnosis Medica, c/Córsega, 345,

08037 Barcelona, Spain

e-mail: jaclavero@yahoo.es dorsiflexion and distraction in the computed tomography scanner. The distance between the anterior border of the inferior tibial articular facet and the posterior border of the anterior tibial artery was measured. The median distance from the anterior border of the inferior tibial articular facet to the posterior border of the anterior tibial artery in ankle dorsiflexion and distraction was $0.9 \mathrm{~cm}$ (range 0.7-1.5) and $0.7 \mathrm{~cm}$ (range $0.5-0.8$ ), respectively. The distance in ankle dorsiflexion significantly exceeded the distance in ankle distraction $(P=0.03)$. The current study shows a significantly increased distance between the anterior distal tibia and the overlying anterior neurovascular bundle with the ankle in a slightly dorsiflexed position as compared to the distracted ankle position. We thereby conclude that the distracted ankle position puts the neurovascular structures more at risk for iatrogenic damage when performing anterior ankle arthroscopy.

Keywords Ankle - Dorsiflexion · Distraction · Anterior ankle arthroscopy

\section{Introduction}

The ankle joint or talocrural articulation at the proximal level is formed by the articular surfaces of the distal tibial and fibular epiphyses and distally by the talus in its superior, lateral and medial aspect (Fig. 1). The morphology of these surfaces forms a hinge-type synovial joint, with a single axis of movement (bimalleolar axis) that allows dorsiflexion (flexion) and plantar flexion (extension) of the ankle and foot in the sagittal plane [15]. Because of this configuration and the fact that it is a load-bearing joint, the intraarticular space is very narrow. In 1931 Burman for this reason concluded that this joint was not suitable for arthroscopy [6]. 


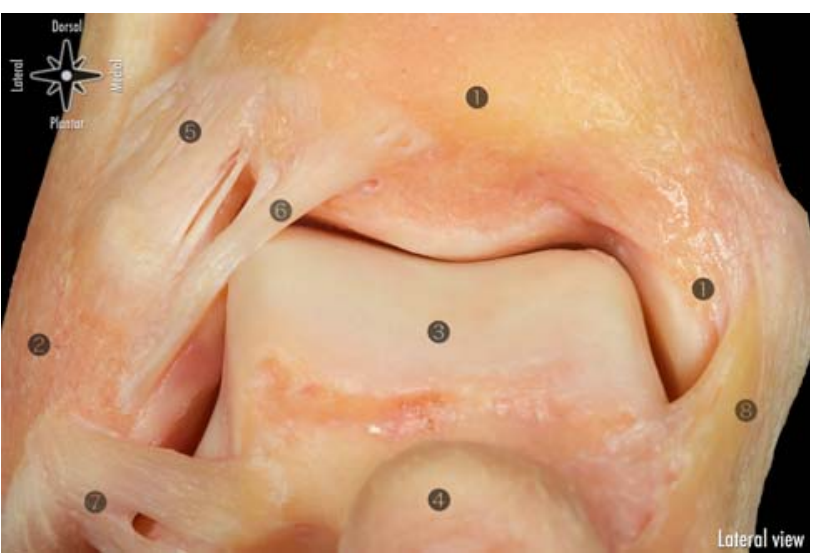

Fig. 1 Anatomic view of the anterior ligaments of the ankle. 1 Tibia and medial malleolus. 2 Lateral malleolus. 3 Talus. 4 Head of the talus. 5 Anterior tibiofibular ligament. 6 Distal fascicle of the anterior tibiofibular ligament. 7 Anterior talofibular ligament. 8 Superficial and deep layers of the medial collateral ligament

In the second half of the last century technical improvements have lead to smaller diameter arthroscopes and improved instruments. These improvements have increased the popularity of the ankle joint arthroscopy. Currently an increasing amount of indications can be diagnosed and arthroscopically treated. Ankle arthroscopy is nowadays considered reliable and relatively safe and is therefore frequently used for treatment of a wide variety of ankle pathology.

Because of the high joint congruency, various methods have been developed to distract the ankle joint with the objective to improve visualization and to obtain access to the articular surface $[2,3,7,8,16,17,19,22,23,26,27$, $29,34-36]$. Fixed joint distraction in combination with a $2.7 \mathrm{~mm}$ diameter arthroscope was popularized by Guhl [16] (Fig. 2). Since the small diameter shaft does not allow for a high volume of water inflow an additional posterolateral portal is usually recommended. This three portal approach was advocated and used for diagnostic as well as therapeutic purposes. A 21-point diagram was introduced by Ferkel and Fischer [11]. This diagram was suggested being able to systematically and reproducibly exam the ankle by arthroscopy from a diagnostic point of view.

With the development and improvement of a variety of additional diagnostics like MRI, CT, and bone scans, the diagnostic use of ankle arthroscopy has been questioned [25]. Especially in the ankle joint a variety of asymptomatic pathological changes, like spurs, ossicles, scar tissue and cartilage damage can coexist. The combination of history, physical examination and additional diagnostics are sufficient for a proper clinical diagnosis and to decide for the best surgical approach. A diagnostic arthroscopy has no added value. Anterior pathology, like impingement syndromes, ossicles, loose bodies and anterior located

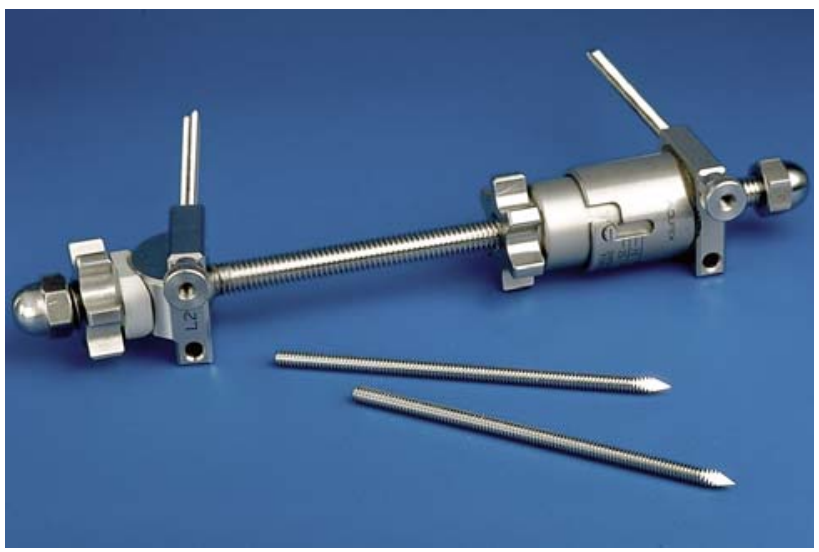

Fig. 2 Invasive distractor for ankle arthroscopy [16]

osteochondral defects can be treated by means of an anterior 2 portal approach [31]. Posterior ankle pathology like the os trigonum, flexor hallucis longus tendinopathy or posterior located osteochondral defects can effectively be treated by a 2 portal hindfoot approach [30, 32].

For the treatment of these lesions routine distraction is not indicated and can even provide a disadvantage since it could reduce the anterior and posterior working area $[10,31]$. For this reason the senior author developed a non distraction method to access the anterior ankle compartment by means of ankle arthroscopy [31]. The key element in this method is to dorsiflex the ankle, thereby opening up the anterior working area, creating sufficient space to use a $4.0 \mathrm{~mm}$ arthroscope (Fig. 3). The large diameter arthroscope allows for an increased saline inflow as compared to the $2.7 \mathrm{~mm}$ arthroscope.

The anterior working area is anteriorly limited by the overlying anatomy which includes the neurovascular bundle (Fig. 4). We hypothesize that in ankle dorsiflexion the anterior neurovascular bundle will move away anteriorly

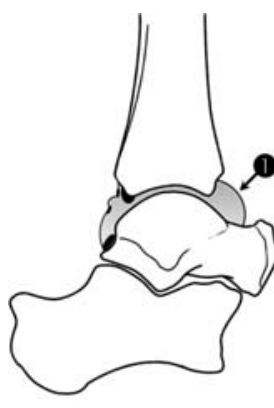

a

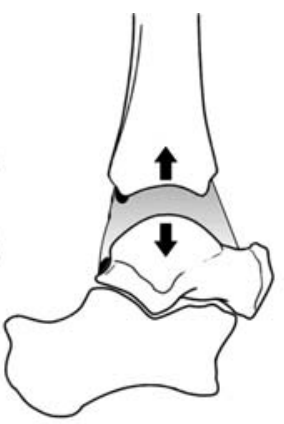

b

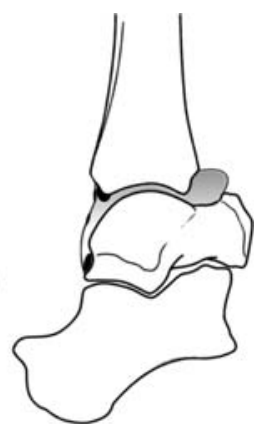

C
Fig. 3 a Schematic view of the ankle joint in the neutral ankle position showing the anterior (1) and posterior working areas. b Interarticular work is possible when distraction (arrows) is used, but the capsular tension reduces the anterior and posterior working areas. c The anterior working area is opened in dorsiflexion of the foot; anterior ankle pathology can easily be treated 


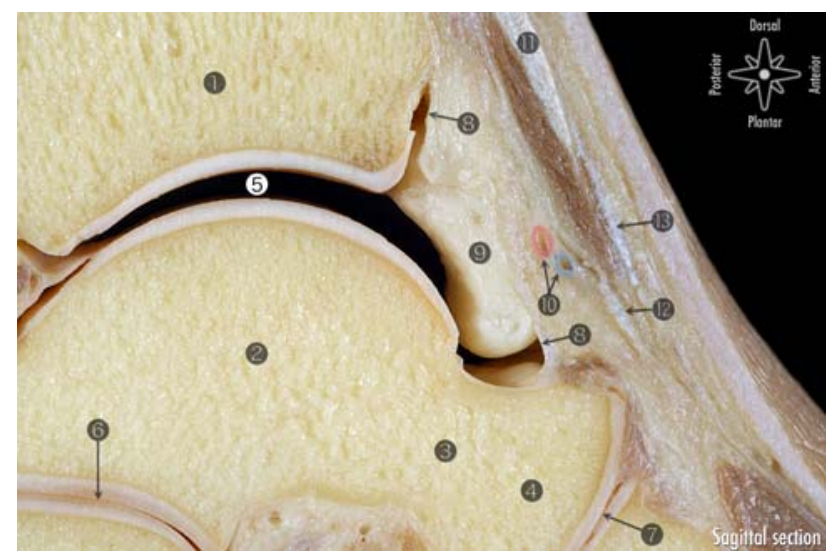

Fig. 4 Sagittal section of the ankle showing the most relevant anatomical structures. 1 Tibia. 2 Talus. 3 Neck of the talus. 4 Head of the talus. 5 Tibiotalar working area. 6 Posterior subtalar joint. 7 Talonavicular joint. 8 Capsule. 9 Intracapsular but extrasynovial fatty tissue. 10 Anterior tibial artery and vein painted with Adobe Photoshop (the deep peroneal nerve has not been identified). 11 Extensor hallucis longus. 12 Deep layer of inferior extensor retinaculum. 13 Superficial layer of inferior extensor retinaculum

from the ankle joint, thereby increasing a safe anterior working area, whereas in ankle distraction the anterior neurovascular bundle is pulled tight towards the joint, thereby decreasing the safe anterior working area (Fig. 3). The objective of this study was to determine the size of the anterior working area for both the dorsiflexion and distraction method.

\section{Materials and methods}

Six ankles from five female and one male fresh frozen specimen, amputated approximately $10 \mathrm{~cm}$ above the knee, from the Department of Pathology and Experimental Therapeutic Unit of Human Anatomy and Embryology in Barcelona, Spain, were examined. The specimens were from the Caucasian origin with a mean age of 81 years (range 79-89 years). Specimens were excluded in case of deformities or scars following ankle surgery and/or limited range of motion, and furthermore ankles with severe atherosclerosis were excluded based on a computed tomography (CT) scan with the arteries containing contrast fluid.

In order to visualize the anterior tibial artery, contrast fluid was injected in the fresh frozen specimens and its orientation relatively to the anterior distal tibia was determined based on CT scans, both in ankle distraction and dorsiflexion. The contrast mix injected into the femoral artery consisted of 1 unity of Barium with 4 unities of commercially available latex and 2 drops of dimeglumine (Magnevist ${ }^{\circledR}$ ) for every $50 \mathrm{ml}$ of solution. The femoral artery was located in the proximal part of the specimen and was separated from the femoral vein. Horizontal incisions in the first and fifth toe were made up to the level of the bone. The femoral artery was first injected with $50 \mathrm{ml}$ water to assess possible vessel obstructions. Subsequently the femoral artery was injected, using a $60 \mathrm{ml}$ filled syringe, with the contrast fluid, until the fluid leaked from the toe incisions.

A special device was developed for reproducible ankle positioning inside the CT scanner (Fig. 5a, b).

The basis of the device is formed by two aluminium sliding arms (A) which are on the left side terminated by a methacrylate block (B). This block is used to fix the proximal part of the specimen to the device using a $12 \mathrm{~mm}$ aluminium pin (C). A movable clamp (D) is attached to the sliding arms and is used to adjust to lower leg length differences. A footplate (E), entirely composed of fibreglass, is attached both to the movable clamp (D) and to a calibrated spring (F). The spring on his turn is attached to another movable methacrylate block $(\mathrm{G})$. Once a specimen correctly fits into the device and the movable clamp (E) is fixed to the aluminium sliding arms, the turning grip $(\mathrm{H})$ is used to vary the distance between the footplate (E) and the aluminium block (G), thereby changing ankle positioning. By increasing this distance the ankle is manipulated into plantar flexion and decreasing the distance results in ankle dorsiflexion.

Furthermore the foot plate can be removed to attach the calibrated spring (F) to a non-invasive foot distracting device. By moving the aluminium block $(G)$ away from the specimen, ankle distraction can be obtained and the amount of force applied can be read from the calibrated spring (Fig 6).

Each specimen was first scanned in slight ankle dorsiflexion followed by ankle distraction, standardized at $100 \mathrm{~N}$, in a sixteen-detector row CT scanner (Aquilion 16; Toshiba Medical, Tokyo, Japan). Scanning was performed using the following parameters, 1-s gantry rotation speed, 0.5-mm slice thickness $(\times 16), 7,5-\mathrm{mm}$ table travel per rotation, X-ray tube voltage was $120 \mathrm{kV}$ and tube current was $150 \mathrm{~mA}$.

The volumetric CT data were reconstructed with a slice width of $10.5 \mathrm{~mm}$ and a reconstruction interval of $0.4 \mathrm{~mm}$. The multiplanar reformatted images and 3-D volume-rendered images were generated on a Vitrea computer workstation (Vitrea version 3.0.1., Vital Images, MN).

The sagittal CT reconstructions were used to assess the anterior working area for both the dorsiflexion and distraction ankle position. A line parallel to the anterior and posterior most distal part of the tibia was drawn to determine the position of the anterior border of the inferior tibial articular facet (Figs. 7, 8). In the sagittal CT reconstruction in which the anterior tibial artery had the widest diameter, the shortest distance between the anterior border of the inferior tibial articular facet and artery was measured using E-film ${ }^{\circledR}$ (Figs. 7, 8). This distance was regarded as the anterior working area. 
Fig. 5 The device is formed by: a Aluminium sliding arm. b Methacrylate block. This block is used to fix the proximal part of the specimen to the device using a aluminium pin c Aluminium pin. d Movable clamp used to adjust to lower leg length differences. e Fibreglass footplate. f Calibrated spring. g Movable methacrylate block. h Grip, used to vary the distance between the footplate (e) and the aluminium block $(\mathrm{g})$
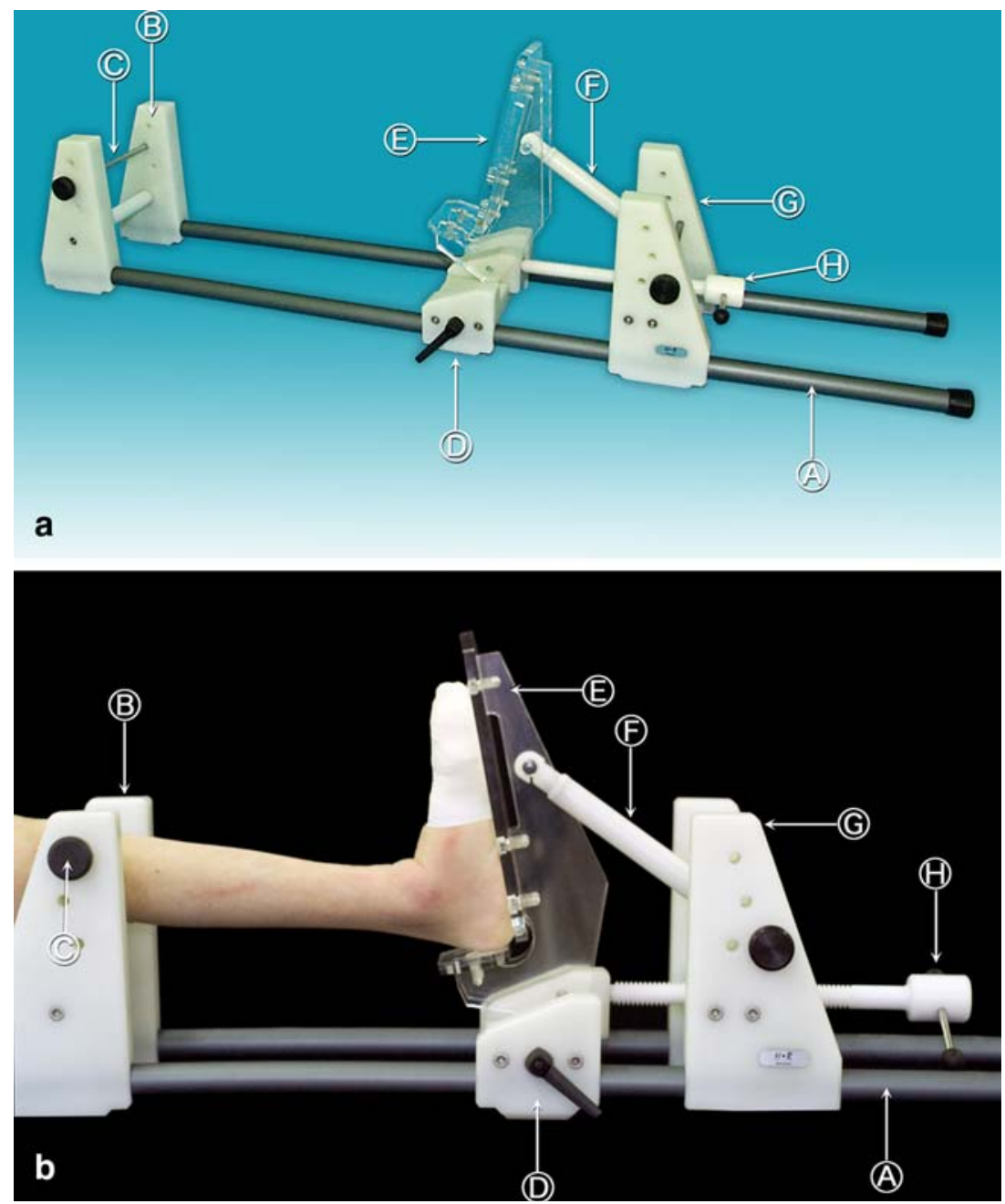

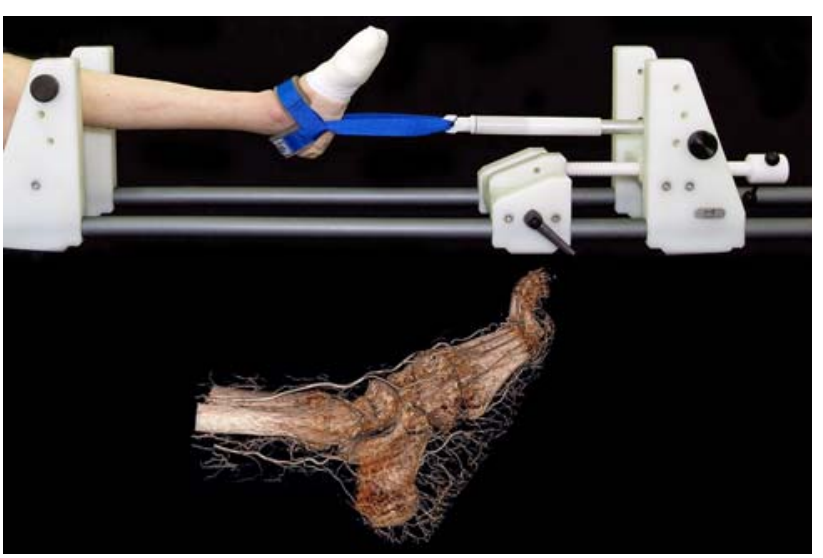

Fig. 6 Example of specimen in the device, using ankle distraction

\section{Statistical analysis}

Each of the measurements was performed twice by two independent observers and inter- and intra-observer reliability was assessed by calculating the intraclass correlation coefficient (ICC). The reliability was considered good if the coefficient exceeded 0.8. Since the sample size was limited, data were presented as medians with accompanying ranges, and a non-parametric Wilcoxon Signed Rank test was performed to investigate whether the anterior working area for the ankle dorsiflexion differed significantly from the ankle distraction $(P<0.05)$.

The amount of dorsiflexion in each of the specimens was not standardized during the CT scanning process but was retrospectively measured by the angle of the footplate as compared to the neutral (90 degree) position. The correlation between the degrees of dorsiflexion applied and the anterior working area was assessed through a Spearman correlation coefficient $(r)$. The correlation was regarded strong if the Spearman coefficient exceeded 0.8.

\section{Results}

None of the specimens met the exclusion criteria, some revealed mild arthrosclerosis of the popliteal artery, which 


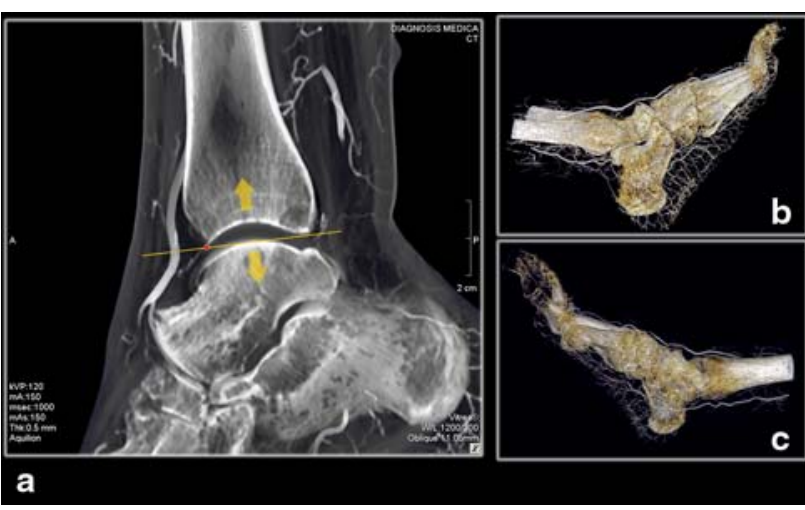

Fig. 7 The sagittal CT reconstructions in the distracted ankle position. A line parallel to the anterior and posterior most distal part of the tibia was drawn to determine the position of the anterior border of the inferior tibial articular facet. The shortest distance between the anterior border of the inferior tibial articular facet and artery was measured using E-film ${ }^{\circledR}$. This distance was regarded as the anterior working area

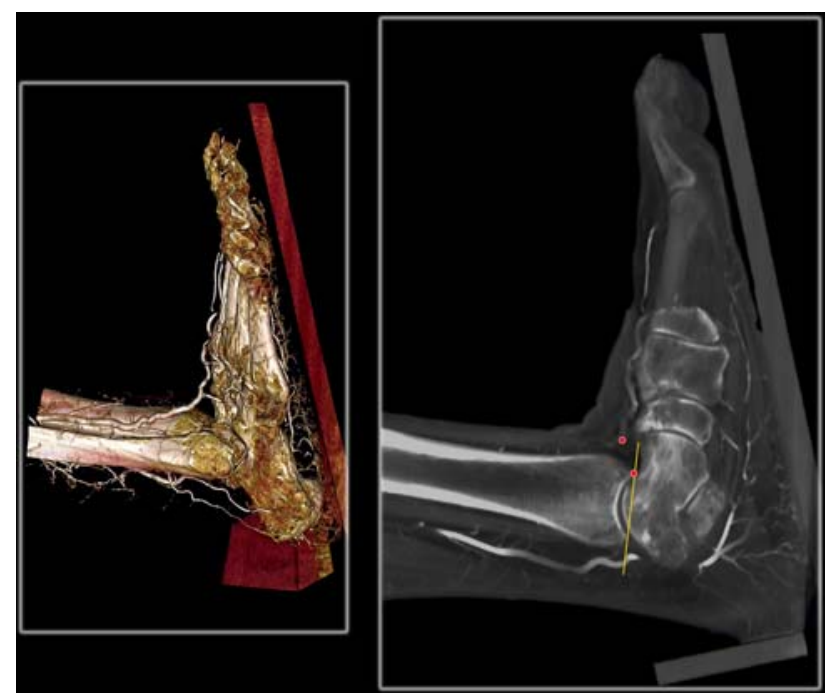

Fig. 8 The sagittal CT reconstructions in ankle dorsiflexion

however, did not interfere with the injected amount of contrast fluid. On average $43 \mathrm{ml}$ contrast fluid was injected in each of the specimens (range 40-50 ml).

Intra-observer reliability coefficient was 0.99 for both observers in the dorsiflexion position and 0.95 and 0.93 in ankle distraction. Inter-observer reliability coefficient was 0.99 for both measurements in ankle dorsiflexion and 0.79 and 0.94 in ankle distraction for the first and second measurement, respectively.

The median distance from the anterior border of the inferior tibial articular facet to the posterior border of the anterior tibial artery (anterior working area) in ankle dorsiflexion and distraction was $0.9 \mathrm{~cm}$ (range 0.7-1.5) and $0.7 \mathrm{~cm}$ (range $0.5-0.8$ ), respectively (Fig. 9). The anterior

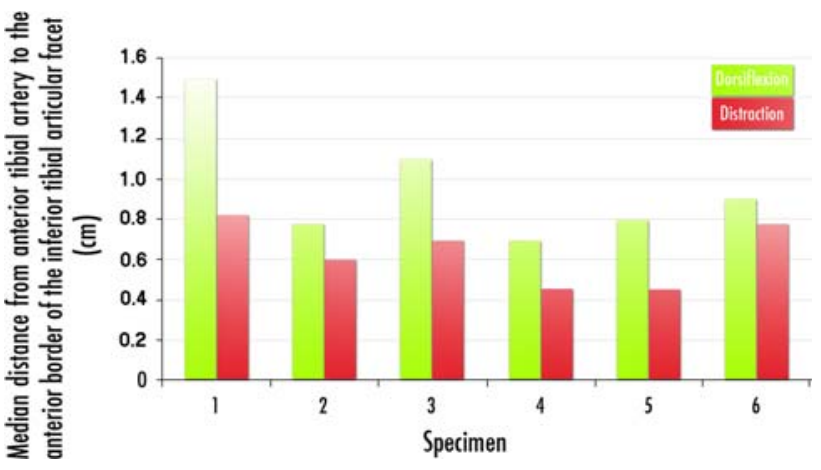

Fig. 9 Median distance from the anterior border of the inferior articular tibial facet to the anterior tibial artery $(\mathrm{cm})$ in ankle dorsiflexion and distraction $(N=6)$

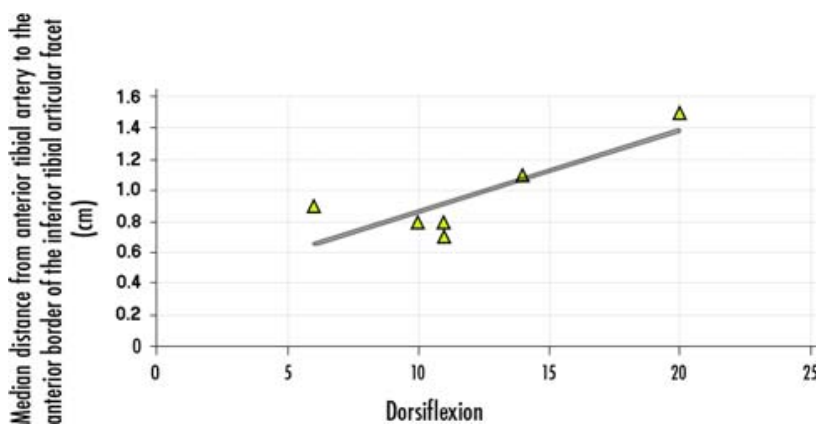

Fig. 10 Correlation between the degrees of ankle dorsiflexion in each specimen and the median distance from the anterior tibial artery to the anterior border of the inferior tibial articular facet $(N=6)$

working area in ankle dorsiflexion significantly exceeded the area in ankle distraction $(P=0.03)$.

The correlation between the degrees of ankle dorsiflexion in each specimen and the median distance from the anterior tibial artery to the anterior border of the inferior tibial articular facet, is shown in Fig. 10. There was no significant correlation between the amount of ankle dorsiflexion and the distance of the anterior tibial artery to the anterior border of the distal tibial facet $(r=0.6$, $P=0.26)$.

\section{Discussion}

The most important finding of this study is that a significantly increased anterior safe working area is created when performing anterior ankle arthroscopy with the dorsiflexion method as compared to the distraction method. With an increased anterior working area, the chance of iatrogenic damage to the neurovascular structures during anterior ankle arthroscopy is decreased. This finding is supported by the reported complication rates for anterior ankle arthroscopy. In ankle arthroscopic series with fixed 
distraction the average complication rate varies between 8 and $17 \%[1,4,5,9,12-14,17,18,20,21,24]$ and is on average $10.7 \%$. A series of 1,305 consecutive arthroscopic procedures with the ankle dorsiflexion method has revealed a complication rate of $3.4 \%$ [33]. This figure compares favorably to the distraction method rates.

The increased working area with ankle dorsiflexion enables the use of a larger diameter arthroscope and arthroscopic instruments. Another advantage is that during ankle dorsiflexion the articular cartilage is protected against iatrogenic damage [28]. The joint is locked in this position which prevents the transfer of loose bodies and/or detached bone fragments from the anterior ankle compartment to posterior. The main advantage of the distraction method is the direct access to the cartilage of the talar dome.

The ankle joint capsule is in close relation to the anterior neurovascular bundle. At the level of the ankle joint, the anterior neurovascular bundle is located just anteriorly to the joint capsule. This bundle is formed by the deep peroneal nerve and the anterior tibial artery or dorsalis pedis artery and veins, depending the level of consideration. We decided to use the anterior tibial artery as a landmark to determine the anterior safety zone.

One of the limitations of our research is that the examined specimens are not comparable to the age group that is normally operated by means of arthroscopy. The mean age at time of death in the examined specimens was 81 years old, whereas ankle arthroscopy is most frequently performed in patients between 18 and 40 years old. Probably with age soft tissue structures, including the anterior neurovascular bundle, become more rigid. The anterior neurovascular bundle will then not move easily with ankle dorsiflexion, resulting in an underestimated distance between the anterior border of the inferior tibial facet and the anterior tibial artery in our study.

Another limitation of the presented research is that during the CT scanning process the joint capsule was not filled with saline, as is during ankle arthroscopy. Saline in combination with ankle dorsiflexion will create a capsular recess, which is probably more prominent as compared to a joint capsule without saline. As the anterior neurovascular bundle will shift with the creation of a prominent capsular recess, in the present report presumably an underestimated safe distance is measured for the dorsiflexion ankle position.

\section{Conclusion}

The current study shows that while performing anterior ankle arthroscopy with the ankle in distraction, the anterior neurovascular structures are more at risk for iatrogenic damage, as compared to anterior ankle arthroscopy with the ankle in dorsiflexion.

Acknowledgments We would like to thank Miguel Rodríguez and Henry Anderson (H-R Fungibles, S.A., Spain) by their effort in the development of the device.

Open Access This article is distributed under the terms of the Creative Commons Attribution Noncommercial License which permits any noncommercial use, distribution, and reproduction in any medium, provided the original author(s) and source are credited.

\section{References}

1. Amendola A, Petrik J, Webster-Bogaert S (1996) Ankle arthroscopy: outcome in 79 consecutive patients. Arthroscopy 12:565-573

2. Andrews JR, Previte WJ, Carson WG (1985) Arthroscopy of the ankle: technique and normal anatomy. Foot Ankle 6:29-33

3. Aydin AT, Ozcanli H, Soyuncu Y, Dabak TK (2006) A new noninvasive controlled intra-articular ankle distraction technique on a cadaver model. Arthroscopy 22:905e1-905e3

4. Barber FA, Click J, Britt BT (1990) Complications of ankle arthroscopy. Foot Ankle 10:263-266

5. Bonnin M, Bouysset M (1999) Arthroscopy of the ankle: analysis of results and indications on a series of 75 cases. Foot Ankle Int 20:744-751

6. Burman MS (1931) Arthroscopy of direct visualisation of joints. An experimental cadaver study. J Bone Joint Surg 13:669-695

7. Cameron SE (1997) Noninvasive distraction for ankle arthroscopy. Arthroscopy 13:366-369

8. Casteleyn PP, Handelberg F (1995) Distraction for ankle arthroscopy. Arthroscopy 11:633-634

9. Cutsuries AM, Saltrick KR, Wagner J, Catanzariti AR (1994) Arthroscopic arthroplasty of the ankle joint. Clin Podiatr Med Surg 11:449-467

10. de Leeuw PA, van Dijk CN (2009) Fixed distraction is not necessary for anterior ankle arthroscopy. Knee Surg Sports Traumatol Arthrosc 17:1399-1400

11. Ferkel RD, Fischer SP (1989) Progress in ankle arthroscopy. Clin Orthop Relat Res 240:210-220

12. Ferkel RD, Heath DD, Guhl JF (1996) Neurological complications of ankle arthroscopy. Arthroscopy 12:200-208

13. Ferkel RD, Small HN, Gittins JE (2001) Complications in foot and ankle arthroscopy. Clin Orthop Relat Res 391:89-104

14. Frey C, Feder KS, DiGiovanni C (1999) Arthroscopic evaluation of the subtalar joint: does sinus tarsi syndrome exist? Foot Ankle Int 20:185-191

15. Golano P, Vega J, Perez-Carro L, Gotzens V (2006) Ankle anatomy for the arthroscopist. Part I: the portals. Foot Ankle Clin $11: 253-273$

16. Guhl JF (1988) New concepts (distraction) in ankle arthroscopy. Arthroscopy 4:160-167

17. Hedley D, Geary NP, Meda P (2001) Ankle arthroscopy: a new technique for non-invasive ankle distraction. Foot Ankle Surg 7:137-139

18. Jerosch J, Schneider T, Strauss JM, Schurmann N (1993) Arthroscopy of the upper ankle joint. List of indications from the literature-realistic expectations-complications. Unfallchirurg 96:82-87

19. Kumar VP, Satku K (1994) The A-O femoral distractor for ankle arthroscopy. Arthroscopy 10:118-119 
20. Lerman BI, Gornish LA, Bellin HJ (1984) Injury of the superficial peroneal nerve. J Foot Surg 23:334-339

21. Martin DF, Baker CL, Curl WW, Andrews JR, Robie DB, Haas AF (1989) Operative ankle arthroscopy. Long-term followup. Am J Sports Med 17:16-23

22. Resch H (1993) Einfache Extensionsbandage fur die Sprunggelenkarthroskopie. Arthroskopie 6:93-94

23. Sartoretti C, Sartoretti-Schefer S, Duff C, Buchmann P (1996) Angioplasty balloon catheters used for distraction of the ankle joint. Arthroscopy 12:82-86

24. Scranton PE Jr, McDermott JE (1992) Anterior tibiotalar spurs: a comparison of open versus arthroscopic debridement. Foot Ankle 13:125-129

25. Stufkens SAS, de Leeuw PA, van Dijk CN (2009) Is there still a place for a diagnostic ankle arthroscopy? Eur Musculoskelet Rev 4:86-94

26. Stutz W (1993) Distraktion des OSG fur arthroskopische Eingriffe mit dem Extensionsshuh. Arthroskopie 6:277-278

27. Takao M, Ochi M, Shu N, Naito K, Matsusaki M, Tobita M, Kawasaki K (1999) Bandage distraction technique for ankle arthroscopy. Foot Ankle Int 20:389-391
28. Tol JL, van Dijk CN (2006) Anterior ankle impingement. Foot Ankle Clin 11:297-310

29. Trager S, Frederick LD, Seligson D (1989) Ankle arthroscopy: a method of distraction. Orthopedics 12:1317-1320

30. van Dijk CN, de Leeuw PA, Scholten PE (2009) Hindfoot endoscopy for posterior ankle impingement. Surgical technique. J Bone Joint Surg Am 91:287-298

31. van Dijk CN, Scholte D (1997) Arthroscopy of the ankle joint. Arthroscopy 13:90-96

32. van Dijk CN, Scholten PE, Krips R (2000) A 2-portal endoscopic approach for diagnosis and treatment of posterior ankle pathology. Arthroscopy 16:871-876

33. van Dijk CN, Van Bergen CJ (2008) Advancements in ankle arthroscopy. J Am Acad Orthop Surg 16:635-646

34. van Dijk CN, Verhagen RA, Tol HJ (2001) Technical note: resterilizable noninvasive ankle distraction device. Arthroscopy 17:E12

35. Waseem M, Barrie JL (2002) A new distraction method in difficult ankle arthroscopy. J Foot Ankle Surg 41:412-413

36. Yates CK, Grana WA (1988) A simple distraction technique for ankle arthroscopy. Arthroscopy 4:103-105 